\title{
Avaliação nutricional do recém-nascido: limitações dos métodos atuais e novas perspectivas
}

\author{
Nutritional assessment of newborn infants: current method limitations and new perspectives
}

Romy Schmidt Brockํ, Mário Cícero Falcão

\section{RESUMO}

Objetivo: Revisar a literatura sobre as abordagens na avaliação nutricional do recém-nascido.

Fontes de dados: Levantamento das publicações sobre avaliação nutricional de recém-nascidos indexadas no $\mathrm{Me}$ dline, SciELO e Lilacs nos últimos dez anos, utilizando os descritores "antropometria" e "avaliação nutricional".

Síntese dos dados: A avaliação nutricional do recémnascido é importante tanto para classificação e diagnóstico de alterações do crescimento intra-uterino, quanto para posterior acompanhamento nutricional e de crescimento. Esta avaliação compreende parâmetros antropométricos, bioquímicos e de composição corpórea. Devido à limitação de métodos laboratoriais e de composição corpórea, a avaliação nutricional do recém-nascido continua sendo baseada em parâmetros antropométricos, como peso, comprimento, perímetros cefálico e braquial e dobras cutâneas. Além dos parâmetros antropométricos, as relações antropométricas são úteis para descrever proporcionalidades corpóreas. Dentre tais relações, destacam-se: razão entre perímetros braquial e cefálico e índice ponderal. O índice de massa corpórea (IMC), bastante utilizado em crianças e adultos como indicador de proporcionalidade do crescimento, não conta com valores de referência para recém-nascidos.

Conclusões: Curvas de IMC para recém-nascidos representariam um complemento na avaliação nutricional neonatal e poderiam proporcionar melhor compreensão do padrão de crescimento intra-uterino e suas variações.

Palavras-chave: avaliação nutricional; antropometria; recém-nascido.

\section{ABSTRACT}

Objective: To review the literature regarding different approaches to the nutritional assessment of the newborn infant.

Data sources: Retrieval of publications on nutritional assessment of newborn infants in Medline, SciELO and Lilacs, during the last ten years using as key-words "anthropometry" and "nutrition assessment".

Data synthesis: The nutritional assessment of the newborn infant is an important tool to diagnose and classify intrauterine growth alterations, and to ensure adequate follow up of growth and nutrition. The assessment includes anthropometric variables, biochemical parameters and body composition evaluation. Due to limitation of laboratorial and body composition methods, the neonatal nutritional assessment is still established by anthropometric variables such as weight, length, brachial and head circumference, and skinfolds. The anthropometric relationships are also useful to describe the body proportionality. Amongst these relations, the most cited are: mid-arm circumference and head circumference ratio and height by weight index. The body mass index (BMI) has been used to assess growth proportionality in children and adults, but there are not reference values for neonates.

Conclusions: Neonatal BMI charts would represent a complement in the neonatal nutritional evaluation and could provide a better comprehension of intrauterine growth and its variations.

Key-words: nutrition assessment; anthropometry; infant, newborn.
${ }^{1}$ Mestre em Ciências da Saúde pelo Departamento de Pediatria da Faculdade de Medicina da Universidade de São Paulo (FMUSP), São Paulo, SP, Brasil

2Doutor em Pediatria pela FMUSP, médico encarregado da Unidade de Cuidados Intensivos Neonatal do Instituto da Criança do Hospital das Clínicas da FMUSP e docente da Universidade Anhembi Morumbi/Laureate Universities, São Paulo, SP, Brasil.
Endereço para correspondência:

Mário Cícero Falcão

Rua Vieira de Moraes, 45, apto. 51

CEP 04617-010 - São Paulo/SP

E-mail: profmariofalcao@yahoo.com.br

Recebido em: 18/7/2007

Aprovado em: 4/10/2007 


\section{Crescimento fetal: fatores moduladores}

O período desde a concepção até o nascimento é caracterizado por impressionante crescimento e desenvolvimento dos tecidos. De uma única célula fertilizada, ocorre a diferenciação em mais de 200 tipos celulares. Isto significa, para o concepto humano, um aumento em comprimento de cerca de 5.000 vezes, em superfície corpórea da ordem de $61 \times 10^{6}$ e em peso de ordem de $6 \times 10^{12(1)}$.

O crescimento do feto está intimamente vinculado à oferta de oxigênio e nutrientes e é influenciado por fatores que atuam em conjunto com um projeto básico determinado pelos fatores genéticos, que são mais importantes no início da gestação, mas sempre influenciados pelo ambiente materno, com maior importância no final da gestação ${ }^{(1)}$.

São atribuídos a fatores genéticos $38 \%$ da variação do peso de nascimento e os $62 \%$ restantes, a fatores não genéticos. Dos fatores não genéticos, metade depende de variáveis maternos e a outra metade, de causas desconhecidas ${ }^{(2)}$.

Os fatores maternos que mais influenciam o crescimento fetal são: paridade, nível socioeconômico, raça, altura, fumo, estado nutricional, fatores relacionados à unidade útero-placentária e hormônios ${ }^{(3)}$. A própria nutrição materna periconceptual influencia a qualidade do crescimento fetal. Estudos que manipularam a nutrição materna em animais de experimentação demonstram que a subnutrição não só altera o crescimento fetal em fases tardias da gestação, como influencia negativamente a regulação hormonal e o peso placentário $^{(3)}$.

Os fatores hormonais desempenham importante papel na regulação do crescimento e desenvolvimento fetal. Eles agem como mensageiros e controlam o desenvolvimento e a diferenciação dos tecidos, de acordo com as condições ambientais existentes para o feto. $\mathrm{O}$ sistema de fator de crescimento insulina-símile (IGF), principalmente o IGF-1 e o IGF-2, participa ativamente no crescimento fetal e placentário no decorrer da gestação(2).

\section{Distúrbios do crescimento intra-uterino}

O estudo dos distúrbios de crescimento intra-uterino e de suas causas permitiu o reconhecimento de situações de restrição ou aceleração de crescimento fetal e, a partir daí, relacioná-las a problemas futuros. Nota-se a importância da classificação dos recém-nascidos quanto à adequação nutricional, a fim de detectar precocemente tais alterações ${ }^{(4)}$.
A partir dessas preocupações, passou-se a entender a diferença entre o recém-nascido prematuro, definido exclusivamente pela idade gestacional, e o de baixo peso devido a problemas de crescimento, ou seja, aquele que sofreu influência de fatores que o afetaram negativamente. Para melhor caracterizar esta problemática, surgiu o termo "restrição de crescimento intra-uterino" (RCIU), que indica que o recém-nascido pequeno para a idade gestacional poderia ter sido maior, caso não houvesse fatores maternos ou fetais que prejudicassem o seu crescimento ${ }^{(5,6)}$, salientando-se que o diagnóstico de restrição do crescimento intra-uterino é obstétrico e, na realidade, o neonatologista diagnostica o recém-nascido pequeno para a idade gestacional.

A definição clássica de RCIU é dada em função do peso e da idade gestacional e refere-se ao recém-nascido cujo peso ao nascer está abaixo do percentil 10 para sua idade gestacional. Esta definição tem sido muito criticada, pois classifica $10 \%$ da população como sendo pequena para a idade gestacional e, portanto, alguns autores preferem utilizar percentis mais baixos de classificação, como o percentil $3^{(7)}$. No entanto, além da importância conceitual, os recém nascidos pequenos para a idade gestacional apresentam maior mortalidade e dificuldade de adaptação neonatal em curto e longo prazo, mostrando que o deficit de crescimento é uma condição patológica ou de doença e, portanto, é adequado manter um maior grau de vigilância ${ }^{(8)}$.

Em contrapartida, também foi observado que os recémnascidos grandes para a idade gestacional, ou seja, que sofreram aceleração do crescimento fetal, apresentam maior morbimortalidade.

O interesse na classificação dos recém-nascidos pelo peso de nascimento e a necessidade de valores de referência de crescimento intra-uterino motivaram a construção de várias curvas de crescimento fetal, com base no peso e na idade gestacional dos recém-nascidos ${ }^{(6,9,10)}$.

\section{Curvas de crescimento}

A avaliação do crescimento fetal tem sido representada por um importante instrumento: as curvas de crescimento intrauterino. Cada uma das curvas foi construída com base em dados antropométricos de amostras populacionais, a partir dos quais foram calculados a média, o desvio padrão (dp) ou os diversos percentis para cada idade gestacional estudada e sexo, com o objetivo de construir um gráfico.

Uma das curvas de crescimento intra-uterino mais conhecida foi publicada por Lubchenco et al, em $1963^{(5)}$, baseada 
em dados de peso de nascimento, idade gestacional, sexo e raça de 5.635 nascidos vivos em Denver, Colorado, Estados Unidos. Essa curva foi construída agrupando-se os recémnascidos de acordo com suas idades gestacionais (de 24 a 42 semanas) e realizada seguindo o critério de percentis, conforme publicação dos mesmos autores em $1966^{(6)}$.

Posteriormente, surgiram outras curvas de crescimento intra-uterino, como a de Usher e McLean, publicada em $1969^{(11)}$, realizada a partir de dados antropométricos de recém-nascidos de Montreal, Canadá, e a de Williams, em $1982^{(12)}$, que utilizaram dados de recém-nascidos de diferentes etnias do estado da Califórnia.

Em nosso meio, Ramos et a ${ }^{(9)}$ elaboraram curvas de crescimento intra-uterino utilizando peso, comprimento e perímetro cefálico de nascidos vivos com idades gestacionais entre 30 e 42 semanas, nascidos entre 1979 e 1982 no berçário anexo à maternidade do Hospital das Clínicas da Faculdade de Medicina da Universidade de São Paulo.

Cada uma destas curvas representa o crescimento intrauterino de uma amostra populacional específica, determinada por variáveis como, por exemplo, grupos étnicos incluídos e altitude do local onde o estudo foi realizado, com restrições para seu uso na população geral.

Com a proposição de estabelecer um padrão de referência para a população dos Estados Unidos, Alexander et al, em $1996^{(10)}$, publicaram uma curva de crescimento intra-uterino com o peso de todos os nascidos vivos do ano de 1991. Foram utilizados dados de mais de 3 milhões de recém-nascidos com idades gestacionais de 20 a 44 semanas para a construção da curva baseada no critério de percentis, diferenciando-se das demais por contemplar crianças bastante imaturas.

Existe o questionamento de que estas curvas não representem verdadeiramente curvas de crescimento fetal, considerando-se que não são realizadas medidas seriadas mas medidas antropométricas pontuais de recém-nascidos de diversas idades gestacionais. Admite-se, portanto, que o peso da criança ao nascer seria o mesmo que ela teria dentro do útero, no mesmo momento, se a gestação continuasse até o termo. É necessário lembrar, entretanto, que o nascimento prematuro se deve a algum estado patológico que levou à interrupção da gestação e, portanto, está associado a alterações fetais, maternas e placentárias que poderiam influenciar negativamente o crescimento intra-uterino ${ }^{(5,9)}$. Apesar de críticas e questionamentos, estas curvas de crescimento continuam sendo amplamente utilizadas para a classificação nutricional dos recém-nascidos.
A importância da criação das curvas de crescimento intrauterino está relacionada à identificação dos recém-nascidos que apresentem desvios de crescimento, tanto para mais, como para menos, e, portanto, com maior morbidade e mortalidade em curto, médio e longo prazo ${ }^{(13)}$.

Em longo prazo, numerosos estudos em todo o mundo indicam uma relação entre baixo peso de nascimento e doenças crônicas na idade adulta. O peso de nascimento - e não apenas a prematuridade - está inversamente relacionado à mortalidade por doença cardiovascular $\mathrm{e}$ síndrome de resistência à insulina, que engloba elevação da pressão arterial, intolerância à glicose e aumento dos níveis séricos de triglicérides (síndrome metabólica) ${ }^{(14)}$. Estas observações têm sido atribuídas à teoria da programação, proposta por Baker et al a partir da década de $1990^{(14)}$, que formularam a hipótese de que a privação nutricional levaria a uma programação metabólica fetal e neonatal, ocorrendo ajustes metabólicos em fases precoces do desenvolvimento para permitir a sobrevivência do feto em circunstâncias inadequadas. Tais ajustes seriam responsáveis por alterações hormonais e metabólicas que resultariam em aumento da resistência à insulina, hipertensão e doenças cardiovasculares na idade adulta ${ }^{(15)}$.

É importante ressaltar que existem curvas de crescimento pós-natal, nas quais estão relacionados o peso e a idade cronológica, sendo úteis para caracterizar a inevitável perda de peso pós-natal de recém-nascidos pré-termos de muito baixo peso. Enfatiza-se que tais curvas não levam em consideração a idade gestacional.

\section{Métodos de avaliação nutricional pós-natal}

A maioria das doenças pode levar a alterações nutricionais. A avaliação do estado nutricional no período neonatal ajuda a detectar algumas doenças ou a inferir o risco de outras, como já foi previamente mencionado. Os objetivos e métodos utilizados para esta avaliação nutricional dependem das circunstâncias em que ela é realizada e, portanto, podem se basear em história clínica, parâmetros bioquímicos, análise da composição corpórea e medidas antropométricas do recém-nascido ${ }^{(16)}$.

\section{Parâmetros bioquímicos}

Pode-se dispor de determinações laboratoriais para a avaliação nutricional do recém-nascido. Dentre estas, destacam-se as mensurações do pool protéico do organismo, como a determinação das proteínas totais e frações ${ }^{(17)}$. 
A determinação da albumina sérica pode ser usada na avaliação nutricional em longo prazo, pois, devido à longa meia-vida, suas concentrações plasmáticas podem ser relativamente poupadas em situações de intenso catabolismo protéico, com diminuição do nível sérico apenas depois de períodos prolongados de oferta inadequada. Ademais, na hipoalbuminemia decorrente de desnutrição, a concentração sérica de albumina se normaliza lentamente após a melhora do aporte nutricional ${ }^{(18)}$.

Dentre os parâmetros bioquímicos que permitem avaliar de forma mais dinâmica o estado nutricional, estão as proteínas séricas de meia-vida curta, como a pré-albumina e a proteína ligada ao retinol, que refletem mais apropriadamente o estado protéico recente e o equilíbrio entre síntese e degradação ${ }^{(19)}$. As massas séricas de pré-albumina e proteína ligada ao retinol se mostram adequadas para a avaliação nutricional seqüencial, por responderem rapidamente a alterações positivas e negativas da oferta nutricional; no entanto, ainda apresentam limitada aplicação no período neonatal, principalmente em recém-nascidos pré-termo. Além disso, a pré-albumina pode sofrer interferências em casos de infecções, uso de corticosteróides e alterações da função hepática. A proteína ligada ao retinol varia de acordo com os níveis séricos de vitamina A e zinco ${ }^{(18,19)}$.

\section{Avaliação da composição corpórea}

Avanços tecnológicos recentes foram responsáveis por um rápido desenvolvimento no campo da avaliação da composição corpórea e deram suporte para novas e poderosas ferramentas de investigação nutricional. A bioimpedância eletromagnética (BIA), a absormetria de raios X duo-energético (DEXA), a tomografia computadorizada e a ressonância magnética são alguns exemplos dos métodos empregados ${ }^{(20-22)}$.

A bioimpedância eletromagnética analisa a quantidade de água corpórea total e a massa magra, com base no princípio de que a condutividade elétrica através de fluidos corpóreos é muito maior em massa corpórea magra do que em massa corpórea com gordura e, a partir destas medidas, estima-se a adiposidade ${ }^{(20)}$.

Em crianças, a bioimpedância eletromagnética é um método inócuo, não invasivo e fácil de usar; no entanto, seus resultados podem ser afetados pela posição do corpo no momento do exame, pelo estado de hidratação, tipo de alimentação recebida, temperatura da pele e atividade física, além de não estar validada no período neonatal ${ }^{(20)}$.

A absormetria de DEXA é um método indireto, de baixa radiação e não invasivo que permite avaliar a gordura corpórea e o conteúdo mineral ósseo em crianças. Atualmente, suas aplicações no período neonatal ainda estão sendo estudadas ${ }^{(21)}$.

Os métodos de imagem, como a tomografia computadorizada e a ressonância magnética, representam um avanço importante no estudo da composição do corpo humano. Em adultos, são considerados métodos de escolha para a determinação de medidas de tecido adiposo e musculatura esquelética e são os únicos capazes de realizar medidas de tecidos e órgãos internos ${ }^{(22)}$.

Chan et al, em $1998^{(22)}$, estudaram o uso da ressonância magnética em crianças para avaliar o tecido adiposo e demonstraram uma correlação positiva com outros índices antropométricos utilizados para determinar a porcentagem de gordura corpórea e a obesidade. No entanto, seu emprego no período neonatal ainda precisa ser mais estudado e sua utilização apresenta implicações práticas, como o transporte do recém-nascido até o aparelho, e econômicas, por seu alto custo.

\section{Parâmetros antropométricos}

A avaliação do estado nutricional no recém-nascido é tarefa complexa, porém de grande importância, tanto na sua classificação e no diagnóstico de alterações do crescimento intra-uterino ${ }^{(4)}$, quanto para posterior acompanhamento nutricional e de crescimento, com o intuito de avaliar a adequação da terapia nutricional instituída ${ }^{(16)}$.

Como já descrito, a avaliação nutricional compreende, além da anamnese e exame físico, parâmetros antropométricos, bioquímicos e avaliação da composição corpórea.

Devido à limitação de métodos laboratoriais e de estudos da composição corpórea, a avaliação do estado nutricional do recém-nascido continua sendo baseada, principalmente, em parâmetros antropométricos, como peso, comprimento, perímetros cefálico e braquial e dobras cutâneas ${ }^{(23)}$.

$O$ peso é a medida antropométrica mais utilizada na avaliação nutricional de recém-nascidos e crianças e está intimamente relacionado ao crescimento. Ao nascimento, varia de acordo com a idade gestacional e em função da quantidade de água corpórea total. O peso é de simples obtenção, não requer equipamento especial e é facilmente reprodutível. Entretanto, a acurácia da medida pode ser prejudicada por materiais ligados ao corpo do recém-nascido no momento da aferição ${ }^{(17)}$.

O comprimento é o melhor indicador de crescimento linear e reflete a massa corpórea magra. É determinado pelo potencial genético do indivíduo e sofre menos influência do meio intra-uterino, por ser poupado em casos de desnutrição 
leve e moderada. A medida acurada do comprimento é mais difícil de ser obtida e deve ser realizada por dois indivíduos, em superfície rígida, com régua apropriada com extremidade cefálica fixa e podálica móvel ${ }^{(17)}$.

O perímetro cefálico apresenta relação direta com o tamanho do encéfalo. É a medida mais poupada em casos de restrição nutricional e, portanto, um indicador menos sensível de desnutrição ${ }^{(17)}$.

A medida do perímetro braquial avalia a massa muscular e a gordura do braço e guarda relação direta com a idade gestacional e o peso ${ }^{(23)}$. É muito usada para a avaliação nutricional, pois se considera que a composição do braço reflita adequadamente a composição corpórea. A medida deve ser realizada no ponto médio, entre o acrômio e o olécrano, procurando-se não enrugar a pele no local. O valor a ser considerado é a média de três medidas consecutivas ${ }^{(17)}$.

Estudos sobre o balanço nutricional demonstram que a estimativa apropriada da quantidade de gordura corpórea total e da porcentagem do peso que equivale à gordura é extremamente importante na determinação da adequada oferta de proteínas e energia para recém-nascidos de diferentes idades gestacionais ${ }^{(23)}$. A medida das pregas cutâneas de forma seriada promove uma estimativa do depósito de gordura e obesidade. No período neonatal, utilizam-se as pregas tricipital, subescapular e bicipital, cujos valores variam de acordo com a idade gestacional ${ }^{(17)}$.

\section{Relações antropométricas}

Além dos parâmetros antropométricos simples, o uso de medidas combinadas ou relações antropométricas são úteis para descrever a composição e a proporcionalidade corpórea, sendo mais acuradas do que medidas isoladas para identificar a morbidade perinatal ${ }^{(23)}$.

A relação entre dois parâmetros antropométricos permite estipular a proporção de crescimento, já que se utiliza a comparação de medidas que são diferentemente afetadas em situações de aceleração e desaceleração do crescimento. Estas relações apresentam uma vantagem baseada no principio fisiológico de que certos compartimentos do corpo são poupados em relação a outros, em casos de desnutrição fetal leve a moderada ${ }^{(23)}$. Citam-se, como exemplos, a relação entre perímetros braquial e cefálico e entre peso e comprimento.

A relação entre os perímetros braquial e cefálico é utilizada como indicador do estado nutricional protéico-calórico e permite, não só identificar os recém-nascidos que apresentaram crescimento intra-uterino inadequado e, portanto, são consi- derados como de maior risco para complicações metabólicas no período neonatal, como também acompanhar a evolução do estado nutricional em recém-nascidos pré-termo ${ }^{(23)}$.

A relação entre peso e comprimento, por sua vez, pode ser determinada pela simples divisão do peso pelo comprimento ou pela razão entre o peso e a potência do comprimento. Para a potência ao cubo, ou seja, o índice ponderal, já existem curvas bem estabelecidas em relação à idade gestacional, sendo inclusive utilizado para determinar a proporcionalidade de recém-nascidos pequenos para a idade gestacional ${ }^{(9)}$. O índice ponderal é uma relação que respeita o volume corpóreo tridimensional e é usado para diferenciar a restrição de crescimento simétrica ou proporcionada da assimétrica ou desproporcionada ${ }^{(6)}$.

A relação entre o peso e o quadrado do comprimento, definida como índice de massa corpórea (IMC) ou índice de Quetelet, criado em 1869, tem sido bastante empregada em crianças e adultos e, recentemente, foi validado para crianças entre zero e 36 meses de idade ${ }^{(24,25)}$. Este índice está sendo amplamente empregado por ser o melhor e mais útil marcador de adiposidade, ou seja, por refletir a proporcionalidade do crescimento. Rolland-Cachera et al, em $1982^{(26)}$, validaram o uso do IMC em crianças como um adequado marcador de adiposidade, demonstrando que este índice, quando comparado a outros, como a relação peso/comprimento e o índice ponderal, apresenta menor correlação com o comprimento e melhor correlação com o peso e pregas cutâneas.

Estudos sobre ganho ponderal também estão utilizando o IMC para comparação e classificação de sobrepeso. Tanaka et al, em $2001^{(27)}$, realizaram um estudo relacionando o peso de nascimento e o IMC no primeiro mês de vida com o mesmo índice aos três anos de vida. Os autores demonstraram que as crianças com maior peso de nascimento e maior ganho ponderal no primeiro mês de vida estão mais propensas a evoluírem com maior comprimento e peso, apresentando risco de obesidade nos meses subseqüentes.

Cole et al, em $1995^{(28)}$, baseando-se em dados da população britânica de 1990, construíram uma curva de IMC do nascimento até os 23 anos de idade, utilizando-se os critérios de percentis. O estudo demonstra a importância dessas curvas de referências de IMC na faixa etária pediátrica, devido às substanciais alterações dos valores deste índice, de acordo com fase de crescimento em que a criança se encontra. Observa-se um aumento importante na primeira infância, seguida de uma queda na idade pré-escolar e um aumento seqüencial mais suave até a idade adulta. 
Karlberg et al, em 2003 ${ }^{(25)}$, construíram uma curva de IMC por idade, em crianças suecas de zero a 24 anos, baseada no critério de média e desvio padrão, procurando complementar os dados anteriores publicados, que se baseavam no critério de percentis.

Em 2000, o National Center for Health Statistics (NCHS) publicou curvas de crescimento revisadas para a população americana, acrescentando curvas de IMC por idade para ambos os sexos, em crianças e adolescentes de dois a $20 \operatorname{anos}^{(29)}$. A utilização destas curvas é recomendada para identificar crianças e adolescentes obesos ou com sobrepeso.

Assim, o IMC tem recebido especial atenção para uso em pediatria. A partir da década de 1990, existem várias recomendações para que este índice seja utilizado rotineiramente na classificação de sobrepeso e obesidade na infância e adolescência.

\section{Considerações finais}

A existência de curvas de IMC para recém-nascidos de diversas idades gestacionais representaria um complemento na avaliação nutricional deste período da vida e poderia proporcionar um elo com as outras curvas de IMC já existentes, estabelecendo, assim, parâmetros de comparação durante todo o crescimento e desenvolvimento do indivíduo ${ }^{(30)}$, além de melhor compreensão do padrão de crescimento intra-uterino e suas variações.

Entretanto, em se tratando de curvas de IMC, poucos são os dados referentes ao período neonatal e, em algumas curvas, quando este grupo foi acrescentado, o número de indivíduos utilizado foi pequeno e não incluiu recém-nascidos com idades gestacionais menores de 35 semanas. Ou seja, faltam pesquisas que analisem o IMC durante o período neonatal, de acordo com as diferentes idades gestacionais, principalmente quando se pretende utilizar esse índice em prematuros.

Com base no exposto, observa-se que o IMC no período neonatal, em diferentes idades gestacionais, ainda não foi explorado. A construção dessas curvas, relacionando o IMC e a idade gestacional, poderá contribuir para melhor avaliar a proporcionalidade corpórea do recém-nascido, ou seja, para analisar a presença de crescimento intra-uterino e neonatal harmônico.

\section{Referências bibliográficas}

1. Brock RS. Valores de referência do índice de massa corpórea para recémnascidos de acordo com a idade gestacional [tese de mestrado]. São Paulo (SP): USP; 2006.

2. Kanaka-Gantenbein C, Mastorakos G, Chrousos GP. Endocrine-related causes and consequences of intrauterine growth retardation. Ann NY Acad Sci 2003;997:150-7.

3. Gluckman PD. Endocrine and nutritional regulation of prenatal growth. Acta Paediatr Suppl 1997;423:153-7.

4. Wilcox AJ. Intrauterine growth retardation: beyond birth weight criteria. Early Hum Dev 1983;8:189-93.

5. Lubchenco LO, Hansman C, Dressler M, Boyd E. Intrauterine growth as estimated from liveborn birth-weight data at 24 to 42 weeks of gestation. Pediatrics 1963;32:793-800.

6. Lubchenco LO, Hansman C, Boyd E. Intrauterine growth in length and head circumferences as estimated from live births at gestational ages from 26 to 42 weeks. Pediatrics 1966;37:403-8.

7. Lee PA, Chernausek SD, Hokken-Koelega AC, Czernichow P, International Small for Gestational Age Advisory Board. International Small for Gestational Age Advisory Board consensus development conference statement: management of short children born small for gestational age, April 24-October 1, 2001. Pediatrics 2003;111:1253-61.

8. Lubchenco LO, Searls DT, Brazie JV. Neonatal mortality rate: relationship to birth weight and gestational age. J Pediatr 1972;81:814-22.
9. Cruz ACS, Falcão MC, Ramos JLA. Análise crítica do uso de curvas de crescimento intra-uterino no período neonatal. Rev Bras Nutr Clin 2006;21:198-203.

10. Alexander GR, Himes JH, Kaufman RB, Mor J, Kogan M. A United States national reference for fetal growth. Obst Gynecol. 1996;87:163-8.

11. Usher R, McLean F. Intrauterine growth of live-born Caucasian infants at sea level: standards obtained from measurements in 7 dimensions of infants born between 25 and 44 weeks of gestation. J Pediatr 1969;74:901-10.

12. Williams RL. Intrauterine growth curves: intra- and international comparisons with different ethnic groups in California. Prev Med 1975;4:163-72.

13. Lubchenco LO, Bard H. Incidence of hypoglycaemia in newborn infants classified by weight and gestational age. Pediatrics 1971;47:831-8.

14. Baker DJ, Gluckman PD, Godfrey KM, Harding JE, Owens JA, Robinson JS. Fetal nutrition and cardiovascular disease in adult life. Lancet 1993;341:938-41.

15. Paneth N, Susser M. Early origin of coronary heart disease (the "Baker hypothesis"). BMJ 1995;310:411-2.

16. Cardoso LE, Falcão MC. Análise do crescimento de recém-nascidos pré-termo de muito baixo peso através de curvas de crescimento pré e pós-natal. Rev Bras Nutr Clin 2006;21:278-83.

17. Falcão MC, Cardoso LE. Avaliação nutricional do recém-nascido pré-termo. Rev Bras Nutr Clin 2001;16:144-7.

18. Cardoso LE, Falcão MC. Nutritional assessment of very low birth weight infants: 
relationships between anthropometric and biochemical parameters. Nutr Hosp 2007;22:322-9.

19. Ambalavanan N, Ross AC, Carlo WA. Retinol-binding protein, transthyretin, and C-reactive protein in extremely low birth weight (ELBW) infants. J Perinatol 2005;25:714-9.

20. Sesmero MA, Mazariegos M, Pedrón C, Jones J, Solomons NW. Bioimpedance electrical spectroscopy in the first six months of life: some methodologic considerations. Nutrition 2005;21:567-73.

21. Braillon PM, Salle BL, Brunet J, Glorieux FH, Delmas PD, Meunier PJ. Dual energy absorptiometry measurement of bone mineral content in newborns: validation of the technique. Pediatr Res 1992;32:77-80.

22. Chan YL, Leung SSF, Lam WW, Peng X, Metreweli C. Body fat estimation in children by magnetic resonance imaging, bioelectrical impedance, skinfold and body mass index: a pilot study. J Paediatr Child Health 1998;34:22-8.

23. Cardoso LE, Falcão MC. Importância da avaliação nutricional de recém-nascidos prétermo por meio de relações antropométricas. Rev Paul Pediatr 2007;25:135-41.
24. Van't Hof MA, Haschke F. Euro-Growth references for body mass index and weight for length. Euro-Growth Study Group. J Pediatr Gastroenterol Nutr 2000;31 (Suppl 1):S48-59.

25. Karlberg J, Kwan CW, Albertsson-Wikland K. References values for change in body mass index from birth to 18 years of age. Acta Paediatr 2003;92:648-52.

26. Rolland-Cachera MF, Sempé M, Guilloud-Bataille M, Patois E, Péquignot-Guggenbuhl F, Fautrad V. Adiposity indices in children. Am J Clin Nutr 1982;36:178-84.

27. Tanaka T, Matsuzaki A, Kuromaru R, Kinukawa N, Nose Y, Matsumoto T et al. Association between birth weight and body mass index at 3 years of age. Pediatr Int 2001;43:641-6.

28. Cole TJ, Freeman JV, Preece MA. Body mass index references curves for the UK, 1990. Arch Dis Child 1995;73:25-9.

29. Kuczmarski RJ, Ogden CL, Guo SS, Grummer-Strawn LM, Flegal KM, Mei Z et al. 2000 CDC growth charts for the United States: methods and development. Vital Health Stat. 2002;246:1-190.

30. Brock RS, Falcão MC, Leone C. Body mass index references values for newborn according to gestational age. Clin Nutr 2004;23:766. 